\title{
Mecanismos de Participación Ciudadana Implementados y Utilizados en el Estado de Nicaragua ${ }^{1}$ \\ Implemented mechanisms of citizen participation and used in the State of Nicaragua
}

\author{
Cristian Rivas-Castillo ${ }^{2}$ \\ iD https://orcid.org/0000-0001-6827-1462 \\ Universidad Politécnica de Nicaragua, UPOLI \\ Karla Rodríguez-Burgos ${ }^{3}$ \\ (iD https://orcid.org/0000-0003-2093-8146 \\ Universidad Autónoma de Nuevo León
}

\section{Como referenciar este artículo:}

Rivas-Castillo, C. \& Rodríguez-Burgos, K. (2020). Mecanismos de Participación Ciudadana Implementados y Utilizados en el Estado de Nicaragua. Revista Ciencia Jurídica y Política, 45-61. Recuperado de: https://portalderevistas.upoli.edu.ni/index.php/5revciencasjuridicasypoliticas/article/view/635

\begin{abstract}
RESUMEN
El presente documento tiene como principal objetivo identificar los mecanismos de participación ciudadana reconocidos por Estado de Nicaragua en este sentido, se establece la forma en como el Estado define y crea estos mecanismos, también, se describe la implementación de los instrumentos existentes en la materia en el país centroamericano, por último, se identifican los casos concretos en los que se haya utilizado alguno de los mecanismos de participación ciudadana. El método aplicado es de corte cualitativo, a través del método jurídico exegético, realizando una revisión de la normativa Nacional con relación al establecimiento de los mecanismos de participación ciudadana, así como la participación de los ciudadanos en los mismos. Se concluye que, aunque existe un marco normativo amplio en materia de participación ciudadana la misma no es acorde a la realidad del país, sin embargo, existe un leve crecimiento en el involucramiento de los individuos en las decisiones públicas a partir de la entrada en vigor de la ley de participación ciudadana del año 2003.
\end{abstract}

Palabras clave: Mecanismo de Participación Ciudadana, Participación Ciudadana, Democracia, Nicaragua, Legislación.

\begin{abstract}
The present document has for as main objective to identify the mechanisms of citizen participation recognized by the state of Nicaragua in that sense, it establishes the way how the state defines and creates those mechanisms. Also, describes the setting of existing instruments in the subject in the American Central country, last, it identifies specific cases which they have been used as a mechanism of citizen participation. The method applied was qualitative, through the exegetic legal method, carrying out a review of the national regulations with relation to the establishment of the mechanisms of citizen participation, as well as their participation. It is concluded that, although there is a normative framework on citizen participation that is not in line to the reality of the country, however, there has been a slight increase in the involvement of individuals in public decisions since the entry of the law on citizen participation in 2003.
\end{abstract}

Key words: Citizen participation mechanism, Citizen, Democracy, Nicaragua Legislation.

\footnotetext{
${ }^{1}$ Este artículo se deriva de los resultados parciales de la investigación Políticas Públicas de Ciencia, Tecnología e Innovación en México, Colombia y Nicaragua financiado por la beca nacional CONACYT en alianza con la Universidad Autónoma de Nuevo León y está asociado al proyecto Diagnóstico de investigaciones, programas, políticas públicas y metodologías de la convivencia escolar en el marco del Programa de convivencia escolar desde perspectivas diagnósticas, preventivas y de intervención, patrocinado por el Facultad de Ciencias Políticas y Relaciones Internacionales de la Universidad Autónoma de Nuevo León en México, Universidad Simón Bolívar y Universidad de Boyacá en Colombia.

${ }^{2}$ Licenciado en Derecho por la Universidad Nacional Autónoma de Nicaragua, Maestrante en Relaciones Internacionales por la Universidad Autónoma de Nuevo León, Becario por el Consejo Nacional de Ciencia y Tecnología (CONACYT, México). Docente Investigador. Castillocris25@yahoo.com

${ }^{3} \mathrm{PhD}$ en Filosofía con Acentuación en Ciencias Políticas. Docente e Investigadora de tiempo completo de la Universidad Autónoma de Nuevo León. Facultad de Ciencias Políticas y Relaciones Internacionales. karoburgos@yahoo.com.mx.
} 


\section{INTRODUCCIÓN}

La participación ciudadana es un derecho reconocido por los Estados que favorece a la generación de capacidades en sujetos y organizaciones comunitarias, con el único objetivo de hacer posible el encuentro de los ciudadanos con sus gobernantes y entre las necesidades de la población y todas aquellas decisiones de la autoridad (Morales, 2015., y Martínez, J., Saldierna, A., Rivera, P. \& Rodríguez-Burgos, K.2017).

En este sentido, se hace evidente la necesidad de la participación directa de la población en la programación, planificación y desarrollo de la vida política, social y económica del país, la cual se debe de encontrar respaldad por instrumentos jurídicos que aseguren y resguarden los derechos fundamentales de los ciudadanos involucrados.

Esta protección del Estado al derecho fundamental de participación es necesario para que los países alcancen una consolidación y desarrollo en términos democráticos. En Nicaragua la participación ciudadana se encuentra protegida y regulada por una variedad de normas jurídicas tales como la Constitución política, la Ley de Municipios y la Ley de participación Ciudadana. Sin embargo, existe un desconocimiento de la sociedad en relación con los mecanismos con los que se cuentan para expresar su opinión o decisión en temas de interés tanto políticos, sociales y económicos. En este sentido, se hace necesario estudiar, identificar y analizar los instrumentos de participación ciudadana con los que cuenta el Estado de Nicaragua.

Por tal razón, la presente investigación tiene como objetivos en primer lugar identificar los mecanismos de participación ciudadana reconocidos e implementados por el Estado de Nicaragua, en segundo lugar, se analizará la forma en como son aplicados dichos instrumentos. 


\section{MARCO TEÓRICO}

\section{Aproximación al concepto de participación ciudadana}

Para Rodríguez-Burgos (2015) la democracia constituye en la actualidad un principio prioritario dentro de la agenda de los Estados, la cual se constituye en su forma de organización política, esta lleva dentro de sus fundamentaciones una serie de premisas básicas, como lo son la protección de la soberanía, renacimiento del pluralismo [tanto político como social], la responsabilidad y limitación de los poderes públicos y el reconocimiento de los ciudadanos en la toma de decisiones de la Administración Pública (Rodríguez-Burgos., Martínez, A., \& Tamez, G. 2015., y Sáenz., Gorjón., \& Rodríguez-Burgos. 2008).

En este sentido, Gil Bravo (2010) establece que la participación es una condición indispensable para el funcionamiento de un Estado democrático, es decir, una sociedad democrática es aquella donde los ciudadanos puedan acceder a los diferentes mecanismos de participación, los cuales pueden ser utilizados para mejorar la vida de las personas dentro de la sociedad.

Por otra parte, para que exista la participación de un individuo dentro de un ordenamiento es necesario que el mismo este dotado de un elemento esencial denominado "Ciudadanía" la cual para Martínez Cárdenas \& Rodríguez-Burgos (2016) es una condición jurídico y político que las personas pueden alcanzar por medio de dos situaciones reconocidas por el Estado, en primer lugar, por medio del nacimiento, en este sentido, se entiende que un individuo es ciudadano de un determinado territorio por el solo hecho de haber nacido dentro del mismo, en segundo, lugar, la ciudadanía se adquiere a través de la naturalización. La ciudadanía es la que permite que a una persona por su condición de miembro de un territorio, nación o Estado le sea otorgado el derecho a participar y decidir de la voluntad general.

Dicho lo anterior, la participación ciudadana es comprendida como el "proceso de aprendizaje y desarrollo tanto personal como colectivo" (EUSKADI, 2014), estos procesos tienen como finalidad buscar la transformación de las relaciones, las respuestas y acciones entre el Estado y la Sociedad, en donde se busca que los jóvenes, las mujeres y demás grupos 
Rivas-Castillo, C. \& Rodríguez-Burgos, $K$.

de personas se involucren activamente para la toma de decisiones de donde habitan (Martínez, A., \& Rodríguez-Burgos. 2017., y Rodríguez-Burgos., Leyva., \& Muñiz. 2014).

Por su parte, en el presente documento se concibe a la participación ciudadana como el conjunto de mecanismos que son utilizados para impulsar el desarrollo político, social y económico de una sociedad (Sáenz \& Rodríguez-Burgos. 2010) fomentando la democracia participativa a través de un elemento esencial como lo es la integración de los individuos en el quehacer político de un determinado Estado.

En este aspecto, la participación ciudadana reconocida e implementada en los Estados modernos están basadas en múltiples mecanismos que permiten el acceso de la ciudadanía en la toma de decisiones de la Administración Pública (Guerrero-Vega, 2020).

\section{Tipos de participación}

En los sistemas democráticos el poder es atribuido a la sociedad en su conjunto, sin embargo, para que este poder sea ejercido es necesario que los ciudadanos tomen parte de las cuestiones o decisiones públicas, en este sentido, la participación se convierte en un elemento esencial dentro de los Estados.

En este orden de ideas, dentro de los Estados existen distintas formas de manifestación de participación, es así, como diversos autores han tratado durante los últimos años identificar los distintos tipos de participación que existen dentro de los sistemas democráticos, en el cuadro número uno se exponen las principales tipificaciones de participación.

En este aspecto, cada uno de los tipos de participación enunciado en la tabla anterior tienen como finalidad hacer posible el involucramiento de los individuos en la vida pública de los Estados, es así, como la participación social hace referencia a las organizaciones o individuos que defienden los intereses de sus integrantes, en este tipo de participación el Estado no es el actor principal sino las instituciones u organizaciones sociales, por su parte, la participación comunitaria es entendida como la búsqueda del bien común de la comunidad mediante la atención de las necesidades básicas de sus miembros. 
Tabla 1. Tipos de participación

\begin{tabular}{ll}
\hline Autor & Tipo \\
\hline Villarreal, 2010 & Participación Social. \\
& Participación Comunitaria. \\
& Participación política. \\
& Participación Ciudadana. \\
Ziccardi,1997 & Participación Institucionalizada. \\
& Participación no Institucionalizada. \\
& Participación autónoma. \\
& Participación clientelista. \\
& Participación Incluyente o equitativa. \\
& Participación política. \\
Ramírez Viveros, 2015 & Participación cívica. \\
& Participación ciudadana.
\end{tabular}

Fuente: elaboración propia, 2020.

Dentro de esta misma clasificación, se encuentra la participación política la cual para Aguilar Villanueva (2006) tiene que ver directamente con el involucramiento del individuo dentro de los mecanismos políticos Estatales tales como; partidos, parlamentos, ayuntamientos, elecciones entro otros, por otra parte, Sánchez Ramos (2009) sostiene que la participación política es una dimensión de la participación ciudadana, debido a que se refiere a la intervención de las personas en la esfera de lo público.

En sentido contrario, la clasificación propuesta por Ziccardi (1997) reconoce la existencia de cinco tipos de participación de los ciudadanos [institucionalizada, no institucionalizada, autónoma, clientelística y participación incluyente o equitativa], en primer lugar, la participación institucionalizada es aquella que se encuentra reglamentada por el Estado para que la ciudadanía pueda participar en los procesos de toma de decisión, es decir, este tipo de participación, es la legalmente autorizada e implementada directamente por los órganos estatales, contrario sensu, la participación no institucionalizada es todo tipo de participación 
no reglamentada, pero que en la práctica es aplicada por los ciudadanos, sin embargo, carece del reconocimiento estatal.

En cuanto, a la participación autónoma se da cuando los individuos participan en la toma de decisiones gubernamentales a través de organizaciones no gubernamentales [ONG], este tipo de participación, al igual que la no institucionalizada no es controlada por el órgano estatal, contrario a este tipo de participación, se sitúa la clientelistica en la cual tanto la autoridad estatal y los individuos se relacionan por medio de intercambio de favores, por último, se encuentra la incluyente o equitativa, en este tipo de participación se promueve la interacción de todos los individuos de una sociedad en los procesos de toma de decisiones sin hacer distinción de colores políticos, religiosos o estrato social (Ziccardi 1997).

Teniendo en cuenta los elementos abordados, en el presente estudio se abordará la participación ciudadana institucionalizada siendo que la misma se encuentra contenida en el marco legal y normativo de la república de Nicaragua. Este tipo de participación ciudadana tiene dos funciones esenciales, en primera instancia, le otorga legitimidad al gobierno, esto como consecuencia de la participación de la mayoría de los individuos en los procesos democráticos, por otro lado, con la participación institucionalizada se promueve una cultura democrática, es decir, al reconocer la autoridad gubernamental estos mecanismos los convierten en parte esencial de la sociedad, como resultado de estas dos situaciones se podrán tener decisiones y gestión pública eficaces.

\section{Instrumentos de la Participación Ciudadana}

Como parte de los instrumentos institucionalizados de la participación ciudadana Villarreal Martínez (2009) identifica dos tipos, aquellos encaminados a la participación individual y los que fomentan la participación colectiva. Los mecanismos individualizados son empleados comúnmente para la orientación de las políticas públicas, también, suelen utilizarse para conformar la agenda de gobierno o bien para la aprobación de cuerpos normativos, dentro de estos instrumentos se pueden encontrar la consulta pública, el referéndum y el plebiscito.

En este sentido, la consulta pública se puede entender como un mecanismo que tiene como finalidad que la sociedad conozca y participe en los procesos de evaluación de los proyectos 
implementados por un Estado, en tal efecto, el gobierno colombiano establece que la consulta pública facilita la interacción continua entre el Estado y los actores regulados, siendo esta una vía para la consolidación de las democracias, lo cual contribuye a evitar la adopción de medidas arbitrarias por parte de los órganos Estatales (Gobierno de Colombia , 2017).

Por otra parte, el referéndum es considerado como un mecanismo de rectificación de las decisiones o actos legislativos, en tal efecto, Aziz (1998) sostiene que este instrumento de participación ciudadana es un contrapeso al poder gubernamental, tal contraposición es dada por el "carácter ratificatorio de los actos normativos" (Wieland Conroy, 2004).

Para terminar, dentro de los instrumentos de participación ciudadana de carácter individual se encuentra el Plebiscito, el cual es conceptualizado como "un mecanismo de consulta directa que hace al pueblo el poder del Estado, para expresar su aceptación o rechazo sobre un asunto en específico de gran importancia para la sociedad” (Rosa Hernández , 2011).

En otro sentido, se sitúan los instrumentos de participación que fomentan el involucramiento de la colectividad, estos mecanismos según Villarreal Martínez (2009) son; la iniciativa ciudadana, los buzones ciudadanos, el monitoreo ciudadano, la contraloría social o ciudadana, los comités y consejos de participación ciudadana.

Por lo que se refiere a la iniciativa ciudadana es considerada un "mecanismo de democracia directa" (Zovatto, 2015), esta permite a los individuos en acción colectiva proponer proyectos de ley, reformas legales o constitucionales, por su parte, los buzones ciudadanos son mecanismos que permiten expresar opiniones, demandas o sugerencias para ser implementadas en las acciones de los gobiernos, en cambio, el monitoreo ciudadano proporciona información en el monitoreo de las políticas públicas implementadas, en este mismo sentido, la contraloría social evaluar y audita estas mimas, por último, los comités y consejos de participación son un mecanismo de participación directa de los actores no gubernamentales en las distintas fases de acción de las decisiones Estatales. 


\section{Marco Normativo de la Participación Ciudadana en el Estado de Nicaragua}

La democracia participativa directa e indirecta es proporcional a la participación ciudadana, esta se entiende no sólo como una forma de gobernar sino como estilo de vida de una determinada sociedad, teniendo en cuenta lo anterior, la constitución política de Nicaragua en su artículo 7 afirma que esta es una "República democrática, participativa y representativa" (Asamblea Nacional , 2014), en tal efecto, a partir de estos tres principios reconocidos en la supra norma nicaragüense se promueve la incorporación de todos los ciudadanos en los espacios de toma de decisión, sin distinción de su clase social, religión o afiliación política, en este aspecto, el articulo 50 expresa que "los ciudadanos tienen derecho a participar en igualdad de condiciones en los públicos y en la gestión estatal" (Asamblea Nacional , 2014).

Por otro lado, la participación ciudadana en el Estado de Nicaragua también se encuentra reconocida en la "Ley de Municipios", en el artículo 3 de la citada normativa establece que es obligación de los gobiernos municipales garantizar la democracia participativa (Ley No.40, 2013), en este mismo sentido, el articulo 36 expresa que “ los municipios promoverán y estimularan la participación ciudadana en la gestión local” esta participación está consagrada en dos mecanismos específicos siendo estos los cabildos municipales y las sesiones de los consejos municipales.

Por último, la Participación Ciudadana en el Estado de Nicaragua se encuentra regulada en la "Ley de participación ciudadana (Ley 475)" norma que tiene como principal propósito "Promover el ejercicio pleno de la ciudadanía en el ámbito político, social, económico y cultural, mediante la creación (...) de mecanismos institucionales que permitan una interacción entre el Estado y la sociedad nicaragüense" (Ley No. 475, 2003). Esta ley es producto de una larga lucha de las organizaciones civiles, Serra Vázquez (2010) expresa que gracias a esta lucha la ley de participación implementada en Nicaragua no se limita a los niveles tradicionales como varios países de la región, sino por el contrario, se trata de una participación que logra alcanzar niveles decisorios tanto en el diseño como en la implementación de políticas públicas. 


\section{Metodología}

El presente artículo, es producto de una investigación de corte descriptivo que tiene como objetivo principal la descripción de algo, generalmente las características o funciones del problema en cuestión, en este tipo de investigaciones no se plantean hipótesis ni predicciones, sino que se limitan a la caracterización del fenómeno estudiado que le interesan al investigador (Tamayo \& Carrillo, 2005)

El enfoque de este estudio es cualitativo, debido a que se investiga el entorno de los individuos de forma holística, donde se estudian los procesos sociales con intervención mínima del investigador, además, se utilizan estrategias flexibles para la obtención de datos (Álvarez-Gayou, 2003).

En este estudio, se utilizó la técnica del análisis de contenido el cual es una técnica utilizada en las investigaciones con enfoque cualitativo, esta técnica es esencialmente utilizada en la interpretación de textos, ya sea escritos, grabados o cualquier otra forma diferente donde puedan existir toda clase de registros.

\section{Análisis}

La participación ciudadana en el Estado de Nicaragua se encuentra sustentada, protegida y reconocida en primera instancia por la Constitución Política específicamente en los artículos 7 y 50, en tal efecto, para dar cumplimiento con lo establecido en los preceptos constitucionales se formuló y promulgo la "Ley de participación ciudadana, ley número 745”, la cual es la encargada de crear y gestionar los mecanismos existente que contribuyen al fortalecimiento de la democracia establecida en los principios Estatales.

En este sentido, la ley de participación ciudadana establece los elementos esenciales de la forma en como esta se implementa en el Estado nicaragüense, en primer lugar, limita el espacio geográfico en que la ley es aplicable siendo estos; nacional, regional, departamental y municipal, también, hace un reconocimiento a cualquier otro mecanismo que sea aplicado por la sociedad aunque estos no se encuentren regulado por la normativa en estudio, así lo expresa el artículo 5 "la presente ley (...) no limita el desarrollo de nuevas formas de 
participación en la vida política, económica, social, cultural, gremial y sindical” (Ley No. 475, 2003).

Por otro lado, la ley establece los principios que serán los ejes rectores de la participación ciudadana en el Estado de nicaragua, por una parte, se establece que la participación es voluntaria, es decir, al ser la participación ciudadana un Derecho Humano la misma no se debe de ejercer por presión o imposición de terceras personas, por otra parte, al ser considerada un Derecho Humano, el órgano legislativo le reconoce el carácter universal, en este aspecto, esta no puede ser limitada por cuestiones de sexo, raza, religión, condición sexual, clase social o distinción política, en este mismo aspecto, se establece que la participación ciudadana es equitativa debido a que proporciona a todos los sectores instrumentos jurídicos que los coloca en un plano de igualdad ante el Estado y la sociedad con la finalidad de mejorar la condición y calidad de vida, por último, se reconoce que el derecho a participar en las decisiones públicas es de carácter pluralista, por lo cual, se debe de proteger el respeto a la diversidad de opiniones que existen dentro de la ciudadanía.

De lo anterior, se desprenden los instrumentos utilizados por el Estado para dar cumplimiento al reconocimiento del derecho de Participación que tienen los ciudadanos nicaragüenses, por tal razón, el artículo 2 de la Ley numero 745 establece que los mecanismos reconocidos son; la iniciativa ciudadana, la consulta ciudadana, las instancias consultivas para la formulación y seguimiento de las políticas púbicas, las asociaciones de pobladores, las organizaciones gremiales, sectoriales, sociales, organizaciones de mujeres y jóvenes en el ámbito local y la consulta ciudadana. También, la ley establece algunos mecanismos que ayudan al perfeccionamiento de los instrumentos utilizados por el Estado, siendo estos; los cabildos municipales, los comités de desarrollo municipal y departamental y los derechos de petición y denuncia ciudadana.

Estos mecanismos reconocidos e implementados por Estado de Nicaragua son aplicados en tres niveles [Nacional, Regional, Local], en la gráfica número 1 se realiza una identificación de los instrumentos utilizados en cada uno de los niveles. 
Grafica 1. Mecanismos Implementados en cada nivel.

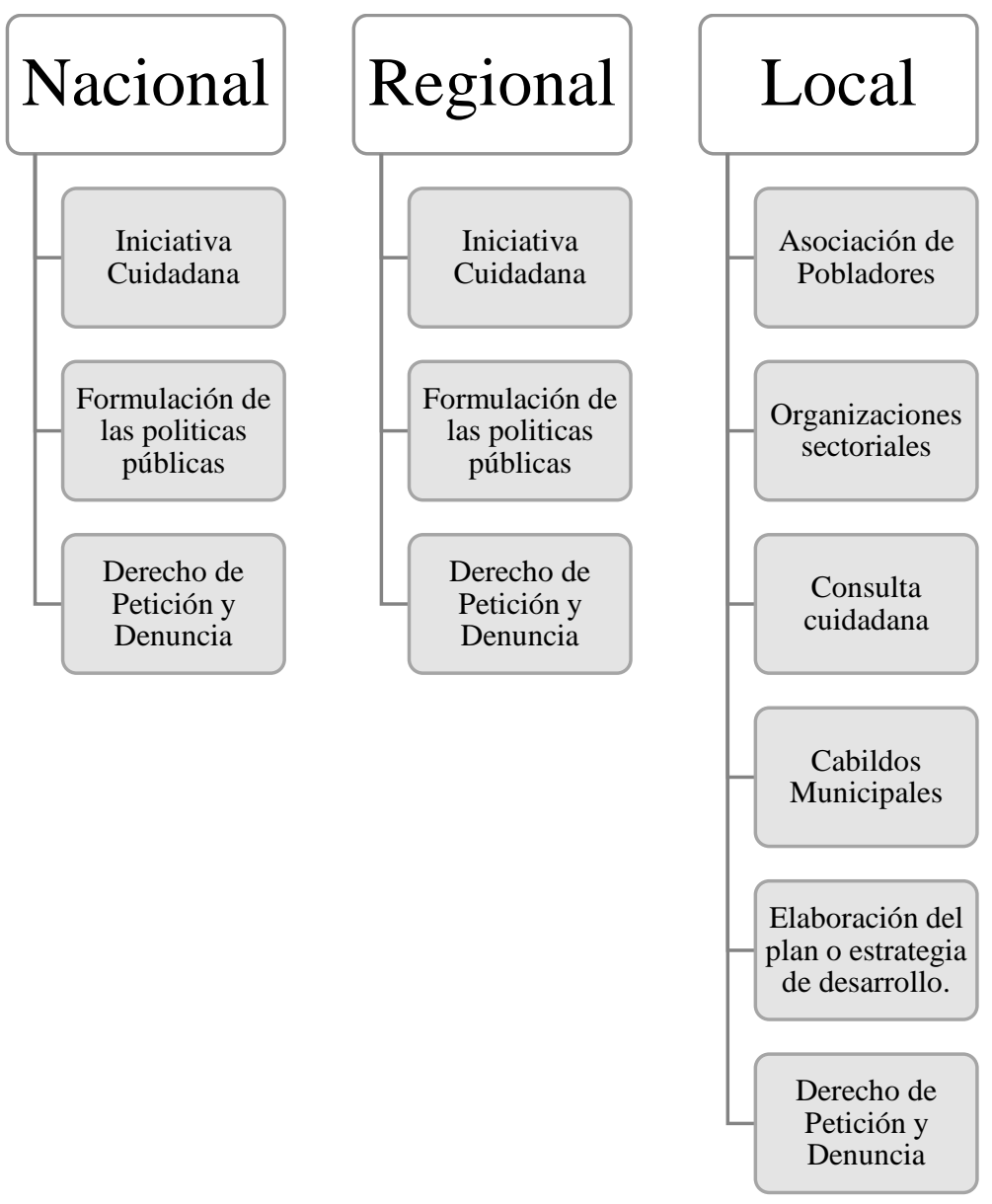

Fuente: Elaboración propia, 2020, en base a lo establecido en la ley “745”

En el primer nivel [Nacional] se reconoce el derecho de todos los ciudadanos a participar en la formación de las leyes [Derecho de Iniciativa], de igual forma, dicho precepto se encuentra regulado en el artículo 140, inciso 4 de la constitución política de la república, sin embargo, este derecho tiene una limitante, siendo esta la imposibilidad de proponer normas en las siguientes materias; leyes orgánicas, leyes tributarias, leyes de índole internacional, leyes de amnistía e indultos, ley general del presupuesto general de la república, leyes de rango constitucional y Constitución política de la República de Nicaragua, códigos de la república y leyes concernientes a la defensa y seguridad nacional.

Desde la entrada en vigor de la ley " 745 " en el Estado de Nicaragua se han aprobado 4 iniciativas propuesta por la ciudadanía, siendo estas; "Ley General de Aguas Nacionales" 
Presentada ante la primer secretaria de la Asamblea Nacional el 11/6/2003 y aprobada el 15/5/2007, "Ley creadora del colegio profesional de medicina y cirugía de Nicaragua" presentada el 5/12/2003 y aprobada el 24/9/2009, "Ley de los Derechos de las personas con Discapacidad" presentada el 25/3/2009 y aprobada el 13/4/2011 y por último la "Ley de pensión reducida por vejez para las personas aseguradas por el instituto Nicaragüense de Seguridad Social” presentada el 15/4/2015 y aprobada el 12/5/2015.

Es necesario mencionar que antes de la entrada en vigor de la ley de participación ciudadana no se aprobó ninguna de las iniciativas presentada por los ciudadanos [se tiene registro de una iniciativa presentada "Ley de aguas Nacionales], es decir, que durante la vigencia la ley 269 la participación ciudadana en la formulación de la iniciativa de ley era prácticamente nula, es a partir del año 2003, fecha que se promulgo la ley "745" que los ciudadanos comenzaron a participar en estos procesos, aunque, en comparación con otros países de la región la participación ciudadana en esta rama es baja (Cerna López , 2013).

Por otro lado, en las regiones autónomas de Nicaragua el Estado reconoce una forma de participación ciudadana en el proceso de formación de la ley distinta a la concebida a nivel nacional, en este sentido, se establece que los ciudadanos de las regiones del país podrán tener incidencia en la formación de normas en las regiones a través del derecho de iniciativa de resolución y ordenanza regional. Esta participación es limitada únicamente a los asuntos concernientes a las necesidades e intereses de las comunidades de los pueblos o comunidades de las regiones autónomas de la Costa Atlántica.

Este derecho de iniciativa de resolución y ordenanza regional reconocido por la ley de participación ciudadana no está permitido ejercerlo en las siguientes materias; ley de autonomía de las regiones de la costa atlántica y su reglamento, el plan de arbitrios de las regiones autónomas, el presupuesto de las regiones autónomas y leyes relativas a defensa de seguridad nacional.

Tras la revisión del sitio web del Consejo Regional Autónomo Costa Caribe Sur [CRACCS] se pudo observar que no existen iniciativas de resolución y ordenanza regional presentadas por personas pertenecientes a la región de la Costa Atlántica (CRACCS, 2020), por lo tanto, 
se establece que la participación ciudadana con relación a la presentación de iniciativas en las regiones autónomas es nula.

Respecto a la participación ciudadana en la formulación de las políticas públicas se reconoce que esta se puede dar en dos ámbitos [Nacional y Regional], la participación a nivel nacional consiste en que los ciudadanos podrán formular en conjunto con las instituciones estatales pertinentes al caso políticas públicas nacionales y sectoriales. Para el caso de las políticas nacionales será coordinada por el Consejo Nacional de Planificación Económica y Social (CONPES), por su parte, las políticas sectoriales serán coordinadas por los Consejos Nacionales Sectoriales, el cual a su vez son coordinados por las instituciones encargadas de la formulación de la política al caso concreto.

En el caso de las Regiones Autónomas, la participación ciudadana en la formulación, diseño e implementación de las políticas públicas se encuentran regias por el Consejo Regional de Planificación Económica y Social [CORPES] de igual manera, en los departamentos y municipios del país existen instituciones encargadas de garantizar la inserción de la población en los asuntos públicos locales en este aspecto, a nivel departamental se han instaurado los Consejos de desarrollo departamental [CDD] y en los municipios se han implementados los Comité de desarrollo municipal [CDM], sin embargo, aunque existen mecanismos reconocidos y regulados por el Estado de Nicaragua la participación en esta área a nivel nacional es escaza.

En tal sentido, los ciudadanos no llegan a tener un rol protagónico en el ciclo de las políticas públicas, a todos los niveles antes mencionados se permite a ciertos grupos sociales tener el derecho a recomendar, coordinar o plantear posiciones con relación a determinadas políticas, limitando de esta manera el derecho a participar en igualdad de condiciones. en este efecto, Serra (2010) afirma que la participación ciudadana en la formulación de las políticas pública en nicaragua son espacios limitados.

De igual forma, se realizó una búsqueda en las distintas plataformas digitales obteniendo como resultado que no existe un sitio web para cada una de las instituciones mencionadas en el párrafo anterior, sin embargo, se pudo observar que este mecanismo reconocido por la ley "745" ha sido complementado por los Consejos y Gabinetes del Poder Ciudadano 
[CPC/GPC], los cuales surgieron como una forma de organización comunitaria bajo el amparo del poder ejecutivo. En tal sentido, el observatorio regional de planificación para el desarrollo de América Latina y el Caribe establece que los CPC/GPC son instancias a las cuales recurre el CONPES para lograr que los ciudadanos den sus opiniones sobre la formulación de políticas o planes gubernamentales (Naciones Unidas, 2020), es decir, son modelos de participación alterno a los establecido en la ley de participación ciudadana. Este modelo ha garantizado la participación de los ciudadanos en la formulación de políticas en temas de salud, educación y en especial en programas como Hambre Cero, Usura Cero y Alimentos para el Pueblo (Almendárez , 2009).

Por último, a nivel Departamental y Municipal la ley de participación ciudadana ha generado como mecanismos de participación: la Asociación de pobladores, organizaciones sectoriales, consulta ciudadana, cabildos municipales, elaboración del plan o estrategia de desarrollo. Las asociaciones de pobladores son comprendidas como organizaciones "comunitarias" (Velásquez-Cruz. 2020) que tienen como objetivo facilitar a los habitantes del municipio la participación en la gestión local (Ley No. 475, 2003).

En Nicaragua se lograron identificar las siguientes asociaciones; Asociación de Pobladores Productores de Miel de Belén [COOAPIR], Asociación de Pobladores del Hormigón Granada, SUKAWALA [Organización Nacional Mayagna], YATAMA [Organización de los pueblos de la madre tierra], TUAHKA [Autodesarrollo de los pueblos indígenas Mayagnas], Asociación Afro Garífuna Nicaragüense, Asociación de Mujeres Indígenas de las Costa Atlántica [AMICA], Consejo de Ancianos de la Nación Comunitaria Moskitia y la Red de Pueblos Indígenas del Pacífico y Centro-Norte de Nicaragua. De lo anterior, se establece que la participación ciudadana por medio de la conformación de asociaciones se da principalmente en las regiones autónomas de Nicaragua.

Otra forma de participación establecida en los municipios y departamentos es a través de las organizaciones sectoriales, las cuales podrán ser de índole cultural, gremial, deportivas, profesionales o de cualquier otra naturaleza, tras la revisión y análisis documental se pudo constatar que no existen registros del total de organizaciones sectoriales existentes en el país. 
Por otro lado, se tiene que la consulta ciudadana es un mecanismo utilizado para conocer la opinión de la comunidad sobre aquellos asuntos que por su naturaleza sean de importancia para los habitantes, en Nicaragua las ultimas consultas ciudadanas registradas han sido; Consulta Ciudadana de la Ley Integral de Promoción del Primer Empleo Juvenil [20092015], Plan Nacional de Desarrollo Humano 2012-2016 [2011], Consulta sobre Seguridad Ciudadana [2012], Plan Nacional de Desarrollo Humanos 2018-2021 [2017] y la Consulta sobre la política "Para una Cultura de Reconciliación y Paz” [2018].

En último lugar, se encuentra como parte de los mecanismos de participación cuidadana los Cabildos Municipales, los cuales son promovidos por cada una de las entidades municipales del país, con el objetivo de promover y estimular la participación de los ciudadanos.

\section{CONCLUSIONES.}

La Participación Ciudadana en el escenario actual "Crisis democráticas" toma una relevancia importante en la agenda de los Estados, en especial en Nicaragua, por tal razón, el Estado se ve en la necesidad de crear y promocionar mecanismos, en tal sentido, se ha construido en las últimas décadas un marco normativo que regule la participación de los ciudadanos en todos los niveles estatales [Nacional, Regional y Municipal], sin embargo, existe una rotura entre lo planteado en las normativas y la realidad de la participación cuidadana, la cual es debido a múltiples factores, como por ejemplo el poco interés de la población en los temas de incidencia política y además la desconfianza por parte de la población hacia las instituciones Estatales.

Sin embargo, existe un leve crecimiento de la participación cuidadana a partir de la entrada en vigor de la Ley “745” ejemplo de esto, son las cuatro iniciativas ciudadanas aprobadas en los últimos años, así como, la participación en la formulación de políticas a través de los Consejos y Gabinetes del Poder Cuidadana.

\section{REFERENCIAS}

Aguilar Villanueva, L. (2006). Gobernanza y gestión pública . FCE. Almendárez, R. S. (2009). Consejos del poder cuidadano y gestión pública en Nicaragua . Managua : EDISA. 
Asamblea Nacional . (2014). Constitución Politica de la República de Nicaragua. Managua, Nicaragua: Gobierno de Nicaragua .

Aziz , A. (1998). Referéndum y Cuidadania . Perfiles Latinoamericanos , 1-25.

Cerna López , M. D. (2013). Participación cuidadana en el proceso de formación de la ley y el derecho de iniciativa. Leon, Nicaragua : UNAN-León.

CRACCS. (Septiembre de 2020). Consejo Regional Autónomo Costa Caribe Sur . Obtenido de https://craccs.gob.ni/

EUSKADI. (2014). Libro blanco de democracia y participación cuidadana . Gobienro Vasco.

Gil Bravo, M. (2010). Participación cuidadana: elemento clave en los procesos de desarrollo de una nación . Enceuntros , 1-8.

Gobierno de Colombia . (2017). Guia para realizar la consulta pública en el proceso de producción normativa. Bogota : Departamento de Planeación.

Guerrero-Vega, R. N. (2020). Modelos de calidad aplicados al servicio de mediación en México. Politica, Globalidad y Ciudadanía, 149-169

Ley No. 475. (22 de Octubre de 2003). Gaceta, Diario Oficial . Ley de Participación Cuidadana . Managua , Nicaragua : Asamblea Nacional .

Ley No.40. (14 de Enero de 2013). La Gaceta,Diario Oficial . Ley No. 40 Ley de Municipios Con Reformas Incorporadas . Managua, Nicaragua: Asamblea Nacional.

Martínez, J., Saldierna, A., Rivera, P. \& Rodríguez-Burgos, K. (2017). Vías de participación ciudadana en un contexto democrático. Editorial Fontamara.

Martínez, A. \& Rodríguez-Burgos, K. (2017). Percepción y participación política de las mujeres en el Estado de Nuevo León, México. En D. Fernández-Matos (Ed.), Liderazgo político y participación política de las mujeres en América Latina en el siglo XXI. (pp. 109-136). Barranquilla: Ediciones Universidad Simón Bolívar.

Morales, R. (2015). La participación cuidadana en la formulaciónde políticas públicas locales, el caso del distrito IV del municipio de Managua (2007-2013). Estudios de Políticas Públicas, 129-151.

Naciones Unidas . (2020). Observatorio Regional de planificación para el desarrollo de América Latina y el Caribe Obtenido de https://observatorioplanificacion.cepal.org/es/instituciones/consejos-y-gabinetesdel-poder-ciudadano-de-nicaragua

Rosa Hernández , G. J. (2011). Formas de participación cuidadana . Cuidad de Mexico : UNAM.

Rodríguez-Burgos, K. (2015). Democracia y tipos de democracia. En X. Arango \& A. Hernández. (Ed.), Ciencia Política. Perspectiva Multidisciplinaria (pp. 49-66). Editorial Tirant lo Blanch.

Rodríguez-Burgos, K., Martínez, A. \& Tamez, G. (2015). Percepción y conceptualización de la democracia actual mexicana. En K. Sáenz, G. Tamez \& K. Rodríguez-Burgos. (Ed.), Sistema Electoral y Político Mexicano (pp. 99-119). Editorial Rechtikal.

Rodríguez-Burgos, K., Leyva, O. \& Muñiz, C. (2014). Participación política y hábitos comunicativos de los jóvenes universitarios en Nuevo León, México. En E. Pastor, G. Tamez \& K. Sáenz (Ed.), Gobernabilidad, ciudadanía y democracia participativa. Análisis comparado España-México (pp. 105-122). Editorial Dykinson.

Sáenz, K., Gorjón, F. \& Rodríguez-Burgos, K. (2008). Democracia, origen y perspectiva. En Democracia y Políticas Públicas (pp. 51-64). Editorial DECISO 
Sáenz, K. \& Rodríguez-Burgos, K. (2010). La promoción de la participación ciudadana. En Elecciones, gobierno y gobernabilidad (pp. 149-176). Instituto Federal Electoral

Sánchez Ramos , M. (2009). La participación cuidadana en la esfera de lo público. Espacios Públicos , 85-102.

Serra Vázquez , L. (2010). Los retos de la participación cuidadana a nivel municipal en Nicaragua . OSAL, 1-19.

Velásquez-Cruz, Lisbeth Carolina. (2020). El facilitador judicial: mediación comunitaria en Nicaragua. Revista Humanismo y Cambio Social. P. 112-119.

Villarreal Martínez , M. T. (2009). Participación Cuidadana y Politicas Públicas . Eduardo Guerra, 31-48.

Wieland Conroy, H. (2004). El referéndum. Concepto general y regulación legal en el Perú. Derecho PUC, 273-302.

Ziccardi, A. (1997). Metodologia de evaluación del desempeño de los gobiernos locales en las cuidades mexicanas . Mexico : UNAM.

Zovatto, D. (2015). Las instituciones de la democracia directa . Revista digital de Derecho Electoral, 34-75. 